\title{
Influence of cross-sectional geometry on the sensitivity and hysteresis of liquid-phase electronic pressure sensors
}

\author{
Yong-Lae Park, ${ }^{1,2, a)}$ Daniel Tepayotl-Ramirez, ${ }^{3}$ Robert J. Wood, ${ }^{1,2}$ and Carmel Majidi ${ }^{3}$ \\ ${ }^{1}$ Wyss Institute for Biologically-Inspired Engineering, Harvard University, Cambridge, \\ Massachusetts 02138, USA \\ ${ }^{2}$ School of Engineering and Applied Sciences, Harvard University, Cambridge, Massachusetts 02138, USA \\ ${ }^{3}$ Department of Mechanical Engineering, Carnegie Mellon University, Pittsburgh, Pennsylvania 15213, USA
}

(Received 14 September 2012; accepted 29 October 2012; published online 8 November 2012)

\begin{abstract}
Cross-sectional geometry influences the pressure-controlled conductivity of liquid-phase metal channels embedded in an elastomer film. These soft microfluidic films may function as hyperelastic electric wiring or sensors that register the intensity of surface pressure. As pressure is applied to the elastomer, the cross-section of the embedded channel deforms, and the electrical resistance of the channel increases. In an effort to improve sensitivity and reduce sensor nonlinearity and hysteresis, we compare the electrical response of $0.25 \mathrm{~mm}^{2}$ channels with different cross-sectional geometries. We demonstrate that channels with a triangular or concave cross-section exhibit the least nonlinearity and hysteresis over pressures ranging from 0 to $70 \mathrm{kPa}$. These experimental results are in reasonable agreement with predictions made by theoretical calculations that we derive from elasticity and Ohm's Law. C 2012 American Institute of Physics. [http://dx.doi.org/10.1063/1.4767217]
\end{abstract}

Soft elastomers embedded with microchannels of liquidphase metal exhibit a unique combination of hyperelasticity and electrical functionalities that allow sensors and circuits to be stretchable, impact-resistant, and mechanically compatible with human tissue and motion. In recent years, researchers have filled thin films of silicone elastomer, such as polydimethylsiloxane (PDMS) or platinum-cured rubber (e.g., EcoFlex ${ }^{\circledR}$ 30; Smooth-On, Inc.), with microchannels of liquid-phase metal alloys (e.g., eutectic gallium-indium, "EGaIn") to produce families of electric circuits and sensors that remain functional even when stretched to greater than three times their natural length. These technologies include stretchable electric wiring, ${ }^{1}$ mechanically tunable antennae, ${ }^{2,3}$ hyperelastic strain sensors, ${ }^{4,5}$ pressure sensors, ${ }^{6-8}$ a wearable keypad, ${ }^{9}$ and sensors that measure bending curvature $^{10}$ and body joint angles. ${ }^{11,12}$

The electrical conductivity of embedded liquid-phase metal channels is strongly influenced by the deformation of the surrounding elastomer. Sensors that measure strain, pressure, and curvature exploit this dependency by mapping elastic deformation with a change in electrical resistance. In the case of pressure sensing, a pressure $p$ applied to the surface of the elastomer will cause the cross-section of the embedded liquid-phase metal channel to deform and the electrical resistance $R$ of the channel to increase. If the channel has a rectangular cross-section, then the resistance scales exponentially with the magnitude of the surface pressure. ${ }^{6}$ While this nonlinear monotonic relationship between $p$ and $R$ is adequate for inferring surface pressure, it requires precise measurement of the electrical resistance-small errors in the measured value of $R$ can lead to dramatic errors in the predicted pressure.

In this manuscript, we introduce alternative cross-sectional geometries that improve the sensitivity of the pressure sensor by reducing the strong nonlinearity between $p$ and $R$. Each

\footnotetext{
${ }^{\text {a) }}$ Author to whom correspondence should be addressed. Electronic mail: ylpark@wyss.harvard.edu.
}

channel has a $0.25 \mathrm{~mm}^{2}$ and is loaded with pressures ranging from 0 to $70 \mathrm{kPa}$. Channels with rectangular or convex crosssectional geometries exhibit a highly nonlinear response with large hysteresis over this range of pressures. In contrast, channels with triangular or concave geometries approach the uniform response of a linear sensor. Not only are these sensors more sensitive at lower pressures, but they also have a greater threshold for measurement errors in $R$ at higher pressures. Moreover, these experimental results are consistent with predictions obtained from a theoretical model based on elasticity and Ohm's Law.

Figure 1 presents four microchannel geometries: (a) square, (b) semi-circle on top of a rectangle, (c) equilateral triangle, and (d) concave triangle. Each channel has a crosssectional area $A_{0}=0.25 \mathrm{~mm}^{2}$ and is filled with EGaIn (resistivity, $\left.\rho=29.8 \times 10^{-8} \Omega / \mathrm{m}\right)^{1}$. Because these channels all share the same initial cross-sectional area $A_{0}$ and length $L=5 \mathrm{~cm}$, they will all have the same initial electrical resistance $R_{0}$ prior to the application of an external surface pressure $p$. As illustrated in Figure 1, the EGaIn-filled microchannels are embedded in a $100 \mathrm{~mm}$ by $100 \mathrm{~mm}$ square elastomer sheet (EcoFlex 30; Smooth-On, Inc., Easton, PA 18042, USA) that is $10 \mathrm{~mm}$ thick. Each sample is fabricated by casting silicone rubber in acrylic molds (see Figure 2) that were produced with a micromilling machine (Microlution 5100-S; Microlution Inc. Chicago, IL 60634, USA). Once the two cured layers, one with a microchannel pattern and the other with no pattern, are prepared, they are bonded by spin-coating the same uncured elastomer between the two layers. Then, EGaIn is injected into the microchannel using hypodermic needles. More details on fabrication of EGaInbased sensors are described in the previous work. ${ }^{6,7}$

Using a commercial materials tester (Instron 5544A, Instron, Norwood, MA 02062, USA), we applied vertical pressure $p$, perpendicular to the top surface of the sample, over a circular area with a $25 \mathrm{~mm}$ diameter. Due to the stiction force of the elastomer to the test plate, the horizontal 

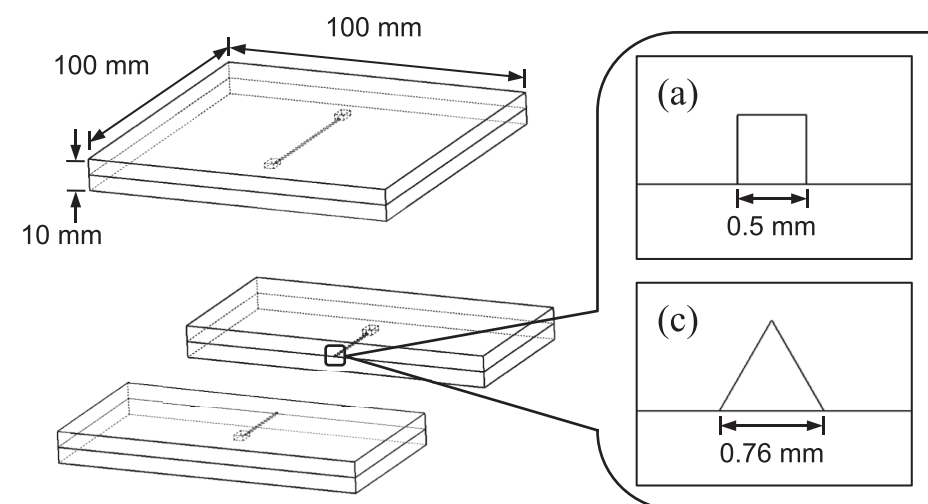

(a)
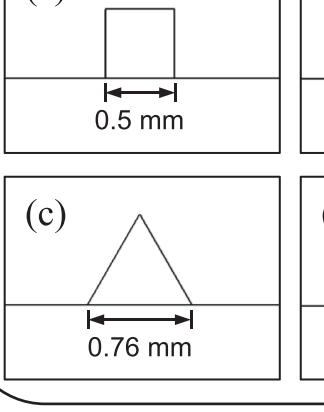
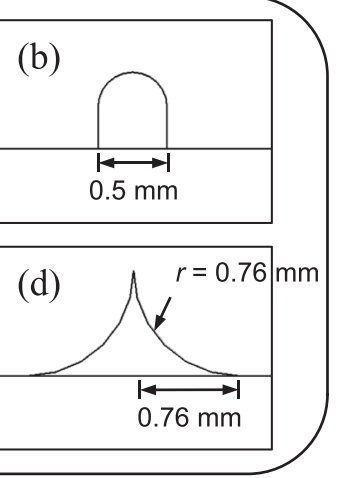

Channel length: $50 \mathrm{~mm}$
FIG. 1. Pressure sensor sample designs with different channel geometries; all four samples have the same crosssectional areas $\left(0.25 \mathrm{~mm}^{2}\right)$. (a) Channel 1: square. (b) Channel 2: semi-circle on top of rectangle. (c) Channel 3: equilateral triangle. (d) Channel 4: concave triangle. motions of the sample were constrained during the tests. Electrical resistance $R$ was simultaneously measured using a precision multimeter (Fluke 8845A, Fluke Corp., Everett, WA 98203, USA) and the relative change in resistance $\Delta R / R_{0}$ was then plotted as a function of $p$, where $\Delta R=R-R_{0}$ represents the total change in resistance. The mean value of the measured initial electrical resistances of the four channels was $0.055 \Omega$ (standard deviation: 0.006) while the theoretically calculated $R_{O}$ was $0.060 \Omega$. According to Ohm's Law, $R=\rho \lambda / A$, where $A$ is the cross-sectional area of the microchannel under an applied pressure $p$. This implies a relative change in electrical resistance of $\Delta R / R_{O}=A_{O} / A-1$, assuming the length change of the microchannel due to the Poisson's ratio of the elastomer is negligible based on the small contact area relative to the entire channel length.

The final cross-sectional area $A=A(p)$ is predicted using approximate theoretical models based on contact mechanics. For the four geometries presented in Figure 1, we estimate ${ }^{6,13}$

$$
\begin{gathered}
A_{a, b}=w\left\{H-\frac{2\left(1-\nu^{2}\right) p w_{a, b}}{E}\right\}, \\
A_{c}=2 H_{c} w_{c}\left\{\frac{1}{2}-\frac{\left(1-\nu^{2}\right) p w_{c}}{E H_{c}}\right\}^{2}, \\
A_{d}=2\left\{\left(r_{d}-\frac{1}{2} \sqrt{2 r_{d} \delta_{d}-\delta_{d}^{2}}\right)\left(r_{d}-\delta_{d}\right)\right. \\
\left.-\frac{1}{2} \arcsin \left(1-\frac{\delta_{d}}{r_{d}}\right) r_{d}^{2}\right\} .
\end{gathered}
$$

Here, $\nu=0.4$ is the Poisson's ratio for the elastomer, $w_{\mathrm{a}, \mathrm{b}}=0.5 \mathrm{~mm}$ is the width of channels 1 and $2, E=125 \mathrm{kPa}$ is the elastic modulus of the elastomer ${ }^{6}, w_{\mathrm{c}}=0.76 \mathrm{~mm}$ and
$H_{\mathrm{c}}=0.66 \mathrm{~mm}$ are the base width and height of channel 3 , $r_{\mathrm{d}}=0.76 \mathrm{~mm}$ is the radius of the circular arcs that form channel 4 , and

$$
\delta_{d}=\frac{4\left(1-\nu^{2}\right) p r}{\pi E} \ln \left(\sqrt{\frac{2 \pi E}{\left(1-\nu^{2}\right) p}}-1\right) .
$$

Equation (1) is based on a linear elastic fracture analysis that treats any channel as a crack that changes height when pressure is applied to the surrounding medium. According to the principle of linear superposition, the change in area $\Delta A$ should be independent of the initial cross-sectional geometry so long as the channel walls do not make contact prior to collapse (i.e., linear superposition cannot account for unilateral contact). In the case of channels 1 and 2, we expect the channel walls to make contact only when the channel has completely collapsed. Therefore, the cross-section area of both channels may be approximate with Eq. (1).

In the case of channels 3 and 4, the sidewall will make contact prior to complete channel collapse. For these channels, we treat the sidewalls as elastic indenters that penetrate the channel base with a depth $\delta$ and a force equal to the vertical component of the pressure exerted above the channel multiplied by the channel width. Treating the side walls of channels 3 and 4 as an elastic wedge and cylindrical indenter, respectively, we use classical solutions in contact mechanics ${ }^{13,14}$ to estimate the penetration of the side walls into the elastic half space below the channel. The areas in Eqs. (3) and (4) are estimated by simply subtracting the area of the original cross-section that is bounded by the channel base and a line that is a distance $\delta$ above the channel base.

Figure 3 shows the experimental results for the pressure response of the four channels. We gradually increased and
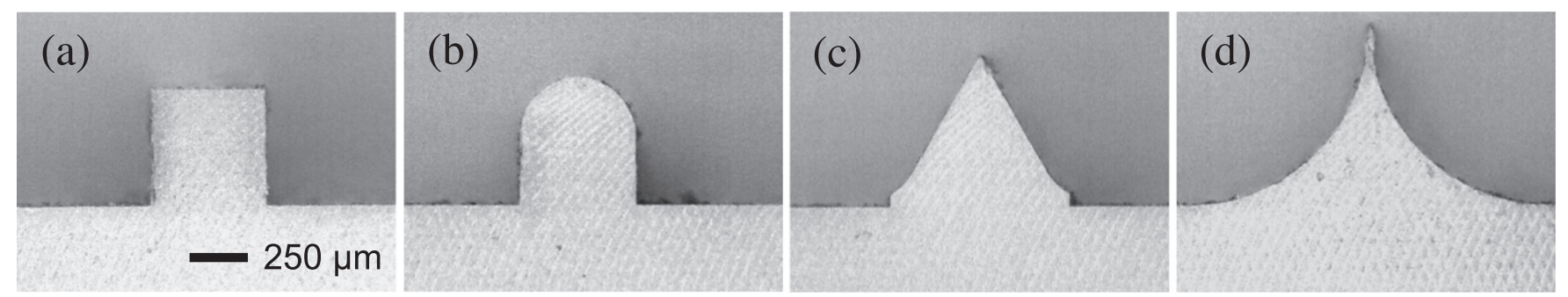

FIG. 2. Photos of the cross-sections of the actual molds with different channel geometries. (a) Channel 1: square. (b) Channel 2: semi-circle on top of rectangle. (c) Channel 3: equilateral triangle. (d) Channel 4: concave triangle. 

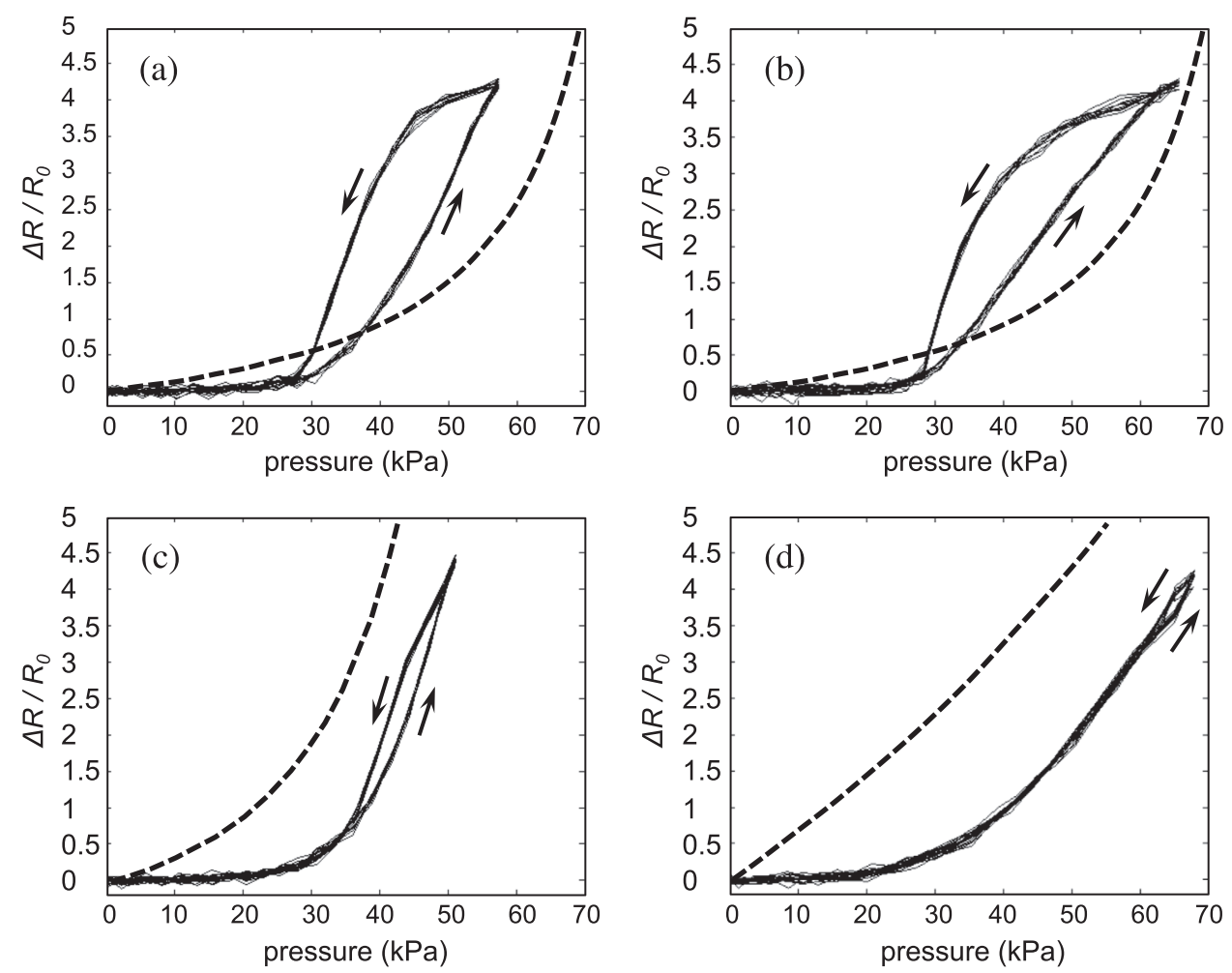

FIG. 3. Experimental results of the resistance changes (solid lines) with a contact pressure range up to $70 \mathrm{kPa}$ and the corresponding theoretical predictions (dotted lines). (a) Channel 1: square. (b) Channel 2: semi-circle on top of rectangle. (c) Channel 3: equilateral triangle. (d) Channel 4: concave triangle. decreased pressure in the range between 0 and $70 \mathrm{kPa}$ with a displacement rate of $2 \mathrm{~mm} / \mathrm{s}$ and measured the corresponding change in electrical resistance. Each channel was tested with 10 repeated trials, and the results are shown as solid lines. The arrows in the plots represent the loading and unloading directions during the tests.

In all cases, we do not measure significant changes in electrical resistance for pressures below $20 \mathrm{kPa}$. Based on the deviations from the mean values, the minimum detectable pressures are $26 \mathrm{kPa}, 27 \mathrm{kPa}, 23 \mathrm{kPa}$, and $20 \mathrm{kPa}$ for channels $1,2,3$, and 4, respectively. Also, we observe significant hysteresis with channels 1 and 2 and negligible hysteresis in channel 4.

Figure 3 also includes theoretical predictions, shown with dotted lines, based on the values of $\Delta A$ obtained from Eqs. (1)-(3). The theoretical derivations do not include viscoelasticity, adhesion between the channel walls or friction within the fluid, or between the fluid and channel walls. Therefore, the theory does not capture the pronounced hysteresis observed with channels 1 and 2 . Nonetheless, it predicts relative changes in electrical resistance that are roughly consistent with the experimental measurements.

As demonstrated in Figure 3, there appears to be reasonable agreement between the experimental measurements and theoretical predictions. Together, the theory and experiment demonstrate that the concave triangular geometry (channel 4) has the most linear response to pressure and exhibits the least amount of hysteresis between loading and unloading. This suggests that microfluidic channels with triangular or concave cross-sections are better suited for soft-matter pressure sensing applications, which require minimal hysteresis and a monotonic electrical response.

Although the theory captures the correct trends and order of magnitude for $\Delta R / R_{0}$, it fails to predict the hystere- sis that we observe between loading and unloading for channels 1 and 2. We expect that this hysteresis may arise from the flow of the liquid-phase alloy into and out of the loaded portion of the microchannel. Compared to channels 3 and 4 , both channels 1 and 2 have a relatively small interfacial area between the fluid and channel walls. In this case, we expect fluid mobility to be predominately controlled by viscosity rather than friction between the fluid and channel walls. During approach, as the loading increases, this viscosity will resist the flow of fluid out of the loaded region. As $p$ increases above $30 \mathrm{kPa}$, the liquid will begin to squeeze out of the channel. During retraction, as pressure is removed, viscosity will resist flow back into the channel leading to a hysteresis in the volume and electrical conductivity of fluid within the loaded portion of the microchannel.

In contrast, fluid in channels 3 and 4 have a relatively large interfacial area with the channel walls. In this case, we expect fluid mobility to be primarily governed by friction between the fluid and channel walls. Strong wetting between the fluid and elastomer will prevent the fluid from squeezing out during loading. This may explain why the theoretical predictions significantly overestimate the relative change in electrical resistance in Figures 3(c) and 3(d). Also, since friction keeps the liquid effectively trapped inside of the channel, we do not expect to see significant hysteresis in the electric response between loading (approach) and unloading (retraction).

Other potential sources of hysteresis include viscoelasticity and stiction between the channel walls. However, viscoelasticity is unlikely to have a primary role since we did not observe significant differences in the electrical response when the samples were loaded at faster or slower speeds. ${ }^{7}$ Stiction between the collapsing channel walls will prevent the fluid from flowing back into the channels after it is squeezed out 
and this would certainly lead to hysteresis. In the case of channels 1 and 2, our theory suggests that the walls only make contact when the channel completely collapses. The absence of an interfacial "crack" may prevent the channel from smoothly opening as pressure is removed, thus, limiting the flow of fluid back into the channel to restore electrical conductivity. In contrast, the theory for channels 3 and 4 suggest that the area of contact between the channel walls increases smoothly during loading. This follows from the assumption that the channel sidewalls behave like elastic indenters that make unilateral contact with the channel base. While adhesion hysteresis is still possible even for smooth and reversible changes in elastic contacts, we expect it to be much less pronounced than in the case of non-smooth contact between the same elastic bodies under similar external pressures.

Lastly, the lower sensor signals in channels 3 and 4 may also be explained by the high surface tension of EGaIn, which may interfere with the ability of the fluid to wet to the sharp corners of the triangular geometries. Instead of being completely filled with EGaIn, channels 3 and 4 may contain voids in the corners of the channel, where, according to the theory, we expect the change in area to be the greatest.

The main contribution of this work is to propose a simple but effective solution to improve sensor signals by changing the physical geometry of embedded microchannels in liquid embedded hyperelastic pressure sensors. The simulation and experiments verified that the geometry of the channel crosssection significantly affects the linearity, sensitivity, and hysteresis in pressure sensing, which are the critical factors to be considered in sensor design. However, the agreement between theory and experiment can be improved with more comprehensive theoretical models that account for fluid viscosity, fluid-wall interactions, and viscoelasticity as well as fluid injection techniques that ensure complete wetting of channel walls.
We used acrylic as a mold material in our experiments due to its short machining time and cost effectiveness. However, metal molds will further improve the surface quality resulting in higher sensor accuracy. Although sensor signals can be post-processed using various types of filters, the approach proposed in this paper could reduce the cost of any necessary signal processing.

This work was supported by the Wyss Institute for Biologically Inspired Engineering at Harvard University, Boston, MA, USA. The authors would like to thank David Breslau for his technical support.

${ }^{1}$ M. D. Dickey, R. C. Chiechi, R. J. Larsen, E. A. Weiss, D. A. Weitz, and G. M. Whitesides, Adv. Funct. Mater. 18, 1097-1104 (2008).

${ }^{2}$ J.-H. So, J. Thelen, A. Qusba, G. J. Hayes, G. Lazzi, and M. D. Dickey, Adv. Funct. Mater. 19, 3632-3637 (2009).

${ }^{3}$ S. Cheng, A. Rydberg, K. Hjort, and Z. Wu, Appl. Phys. Lett. 94, 144103 (2009).

${ }^{4}$ H.-J. Kim, C. Son, and B. Ziaie, Appl. Phys. Lett. 92, 011904 (2008).

${ }^{5}$ S. Cheng and Z. Wu, Adv. Funct. Mater. 21, 2282-2290 (2011).

${ }^{6}$ Y.-L. Park, C. Majidi, R. Kramer, P. Berard, and R. J. Wood, J. Micromech. Microeng. 20, 125029 (2010).

${ }^{7}$ Y.-L. Park, B. Chen, and R. J. Wood, IEEE Sens. J. 12, 2711-2718 (2012).

${ }^{8}$ R. D. P. Wong, J. D. Posner, and V. J. Santos, Sens. Actuators, A 179, 62-69 (2012).

${ }^{9}$ R. Kramer, C. Majidi, and R. J. Wood, in Proceedings of IEEE International Conference on Robotics and Automation, Shanghai, China, 9-13 May 2011 (IEEE, 2011), pp. 1103-1107.

${ }^{10}$ C. Majidi, R. Kramer, and R. J. Wood, Smart Mater. Struct. 20, 105017 (2011).

${ }^{11}$ R. Kramer, C. Majidi, R. Sahai, and R. J. Wood, in Proceedings of the IEEE/RSJ International Conference on Intelligent Robots and Systems, San Francisco, USA, 25-30 September 2011 (IEEE, 2011), pp. 1919-1926.

${ }^{12}$ Y.-L. Park, B. Chen, D. Young, L. Stirling, R. J. Wood, E. Goldfield, and R. Nagpal, in Proceedings of the IEEE/RSJ International Conference on Intelligent Robots and Systems, San Francisco, USA, 25-30 September 2011 (IEEE, 2011), pp. 4488-4495.

${ }^{13}$ I. N. Sneddon, Fourier Transforms, 1st ed. (McGraw-Hill, New York, 1951).

${ }^{14}$ K. R. Schull, Mater. Sci. Eng. R 36, 1-45 (2002). 